Ralf Meyer

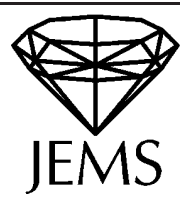

\title{
Excision in entire cyclic cohomology
}

This article is a part of my thesis written at the Westfälische Wilhelms-Universität Münster under the direction of Prof. Dr. Joachim Cuntz.

Received December 16, 1999 / final version received April 18, 2001

Published online May 29, 2001 - (c) Springer-Verlag \& EMS 2001

\begin{abstract}
We prove that entire and periodic cyclic cohomology satisfy excision for extensions of bornological algebras with a bounded linear section. That is, for such an extension we obtain a six term exact sequence in cohomology.
\end{abstract}

\section{Introduction}

A convex vector bornology on a vector space is a collection of subsets satisfying some conditions [8]. A typical example is the collection of bounded subsets of a locally convex vector space. A bornological algebra is a (possibly non-unital) algebra with a bornology for which the multiplication is bounded. Following Cuntz and Quillen [5], we define the entire cyclic cohomology $\mathrm{HE}^{*}(A)$ and the periodic cyclic cohomology $\mathrm{HP}^{*}(A)$ of a bornological algebra $A$ using the $X$-complex $X(\mathcal{T} A)$ of the tensor algebra $\mathcal{T} A$ of $A$. We furnish $X(\mathcal{T} A)$ with a certain bornology and define $\operatorname{HE}^{*}(A)$ as the homology of the complex of bounded linear maps $X(\mathcal{T} A) \rightarrow \mathbb{C}$. This definition generalizes Connes's original definition of entire cyclic cohomology for locally convex algebras [1]. Our main result is the following theorem:

Theorem 1. Let

$$
0 \longrightarrow K \stackrel{i}{\longrightarrow} E \stackrel{p}{\longrightarrow} Q \longrightarrow 0
$$

be an extension of bornological algebras with a bounded linear section $s: Q \rightarrow E$. Thus $E \cong K \oplus Q$ as bornological vector spaces.

Then there is a natural exact sequence

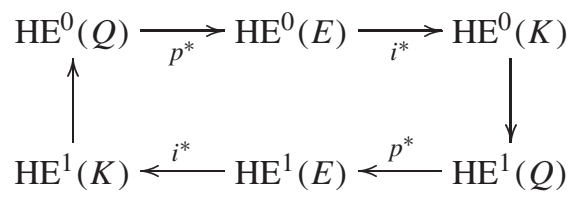

and a similar exact sequence for periodic cyclic cohomology $\mathrm{HP}^{*}(\sqcup)$.

R. Meyer: SFB 478 - Geometrische Strukturen in der Mathematik, Westfälische WilhelmsUniversität Münster, Hittorfstrasse 27, 48149 Münster, Germany

e-mail: rameyer@math.uni-muenster.de

Mathematics Subject Classification (2000): 19D55, 18G60 
Cuntz and Quillen [6], [3] prove excision for the (bivariant) periodic cyclic cohomology of algebras without additional structure and certain topological algebras. In a critical step of their argument they use methods of Wodzicki [12] that are difficult to adapt to entire cyclic cohomology. Recently, Puschnigg has been able to do this [11]. However, his proof of excision works only for the special class of algebras that are inductive limits of Banach algebras. We will prove excision without resorting to Wodzicki's methods.

Let $X(\mathcal{T} E: \mathcal{T} Q)$ be the kernel of the map $X(\mathcal{T} p): X(\mathcal{T} E) \rightarrow X(\mathcal{T} Q)$ induced by $p$. The excision theorem amounts to the assertion that the complexes of bornological vector spaces $X(\mathcal{T} K)$ and $X(\mathcal{T} E: \mathcal{T} Q)$ are homotopy equivalent. Our key idea is to use the left ideal $\mathcal{L}$ in $\mathcal{T} E$ generated by $K$. In the first part of the proof, we show that $X(\mathcal{T} E: \mathcal{T} Q)$ and $X(\mathcal{L})$ are homotopy equivalent. This is done by homological algebra: We have to verify that certain $\mathcal{L}$-bimodules are free and to compute their commutator quotients. In the second part of the proof, we show that $X(\mathcal{T} K)$ and $X(\mathcal{L})$ are homotopy equivalent. This follows from the homotopy invariance of the $X$-complex, once we have a bounded splitting homomorphism $v: \mathcal{L} \rightarrow \mathcal{T} \mathcal{L}$ for the natural projection $\mathcal{T} \mathcal{L} \rightarrow \mathcal{L}$. To construct $v$, we write down a bilinear map $E \times \mathcal{T} \mathcal{L} \rightarrow \mathcal{T} \mathcal{L}$ and use the universal property of $\mathcal{T} E$. The main difficulty is to show that $v$ is bounded.

Periodic and entire cyclic cohomology are special cases of corresponding bivariant homology theories. We actually prove excision for these bivariant theories, in both variables. Furthermore, the argument below can be used to prove excision for $\mathrm{HP}^{*}$ in various categories of algebras: topological algebras, bornological algebras, algebras over a commutative ground field of characteristic zero, etc. We do not pursue these generalizations because our presentation is optimized for the entire theory. The results about $\mathrm{HP}^{*}$ that we prove require only little extra work.

\section{Tensor algebras, filtrations, and periodic cyclic cohomology}

We recall the Cuntz-Quillen approach to periodic cyclic cohomology [5].

Let $A$ be an algebra, possibly without unit. Let $A^{+}$be the algebra obtained by adjoining a unit to $A$. As a vector space, $A^{+}=A \oplus \mathbb{C}$. Let $\Omega^{0} A:=A$, $\Omega^{n} A:=A^{+} \otimes A^{\otimes n}$ for $n \geq 1$, and $\Omega A:=\sum_{n \geq 0} \Omega^{n} A$. We endow $\Omega A$ with the usual $\mathbb{Z} / 2$-grading, multiplication, and differential $d$ as defined in [2]. The multiplication of differential forms yields a natural $A$-bimodule structure on $\Omega^{n} A$ for all $n$.

Let $\Omega^{\text {even }} A$ and $\Omega^{\text {odd }} A$ be the even and odd part of $\Omega A$. Let $\gamma=$ id on $\Omega^{\text {even }} A$ and $\gamma=-$ id on $\Omega^{\text {odd }} A$. The Fedosov product on $\Omega A$ is defined by

$$
x \odot y:=x \cdot y-d \gamma(x) \cdot d y .
$$

That is, $x \odot y=x y-d x d y$ if $x$ is even and $x \odot y=x y+d x d y$ if $x$ is odd. The Fedosov product is associative. We call $\mathcal{T} A:=\left(\Omega^{\text {even }} A, \odot\right)$ the tensor algebra of $A$. It has the following universal property [4]. A linear map $l: A \rightarrow B$ into an algebra $B$ can be extended uniquely to a homomorphism $f: \mathcal{T} A \rightarrow B$. In terms 
of the curvature $\omega_{l}\left(x_{1}, x_{2}\right):=l\left(x_{1} \cdot x_{2}\right)-l\left(x_{1}\right) \cdot l\left(x_{2}\right)$ of $l$, we have

$$
f\left(\left\langle x_{0}\right\rangle d x_{1} \ldots d x_{2 n}\right)=l\left\langle x_{0}\right\rangle \cdot \omega_{l}\left(x_{1}, x_{2}\right) \cdots \omega_{l}\left(x_{2 n-1}, x_{2 n}\right)
$$

for all $\left\langle x_{0}\right\rangle \in A^{+}, x_{1}, \ldots, x_{2 n} \in A, n \geq 0$. The expression $\left\langle x_{0}\right\rangle$ should remind you of the notation for optional arguments in computer handbooks. Thus $\left\langle x_{0}\right\rangle$ is either $x_{0} \in A$ or just missing. In the latter case, it behaves like $1 \in A^{+}$and $l\left\langle x_{0}\right\rangle=1 \in B^{+}$.

The commutator quotient $V /[]=,V /[V, A]$ of an $A$-bimodule $V$ is defined as the cokernel of the map $A \otimes V \rightarrow V, a \otimes x \mapsto a x-x a$. Let $X_{\beta}(A)$ be the complex

$$
0 \longrightarrow \Omega^{1} A /[,] \stackrel{b_{*}}{\longrightarrow} \Omega^{0} A \longrightarrow 0
$$

with $A$ in degree $0, \Omega^{1} A /[$,$] in degree 1$, and $b_{*}\left(\left\langle x_{0}\right\rangle d x_{1} \bmod [],\right):=\left\langle x_{0}\right\rangle x_{1}-$ $x_{1}\left\langle x_{0}\right\rangle$ for all $\left\langle x_{0}\right\rangle \in A^{+}, x_{1} \in A$. The $X$-complex $X(A)$ of $A$ is obtained by adding another boundary $d_{*}: A \rightarrow \Omega^{1} A /[],, x \mapsto d x \bmod [$,$] to the definition of X_{\beta}(A)$.

The universal property implies that the tensor algebras $\mathcal{T} A$ and $\mathcal{T} B$ are isomorphic if $A$ and $B$ are isomorphic as vector spaces, that is, have the same dimension. Thus the naked complex $X(\mathcal{T} A)$ cannot contain any interesting information. We can, however, encode interesting homological information in $X(\mathcal{T} A)$ if we endow it with additional structure. We are going to define a filtration and a bornology on $X(\mathcal{T} A)$ that encode the periodic and the entire cyclic cohomology of $A$, respectively.

A filtration on a vector space $V$ is a decreasing sequence of subspaces $\left(V_{n}\right)$. Usually, we have $\bigcap V_{n}=\{0\}$ but it is not useful to require this. A filtered vector space is a vector space $V$ furnished with a filtration $\left(V_{n}\right)$. It gives rise to a projective system of vector spaces $\left(V / V_{n}\right)_{n \in \mathbb{N}}$. A linear map $f: V \rightarrow W$ between filtered vector spaces is called filtered iff it descends to a morphism of projective systems $\left(V / V_{n}\right) \rightarrow\left(W / W_{n}\right)$. That is, for all $n \in \mathbb{N}$ there is $m \in \mathbb{N}$ such that $f\left(V_{m}\right) \subset W_{n}$. A filtered isomorphism is a filtered linear map with a filtered two-sided inverse.

Let $V$ and $W$ be filtered vector spaces. We define canonical filtrations on the direct sum $V \oplus W$ and the tensor product $V \otimes W$ by $(V \oplus W)_{n}:=V_{n} \oplus W_{n}$ and $(V \otimes W)_{n}:=V_{n} \otimes W+V \otimes W_{n}$. Thus $V \oplus W /(V \oplus W)_{n} \cong\left(V / V_{n}\right) \oplus\left(W / W_{n}\right)$ and $V \otimes W /(V \otimes W)_{n} \cong\left(V / V_{n}\right) \otimes\left(W / W_{n}\right)$. A bilinear map $V \times W \rightarrow \sqcup$ is filtered iff the induced linear map $V \otimes W \rightarrow \sqcup$ is filtered. A filtered algebra is a filtered vector space with a filtered associative multiplication.

We filter a subspace $X \subset V$ by $X_{n}:=V_{n} \cap X$ and the quotient $V / X$ by $V_{n} \bmod X$. Each vector space $V$ carries a trivial filtration defined by $V_{n}:=\{0\}$ for all $n$. We always endow $\mathbb{C}$ with the trivial filtration.

The Hodge filtration on $\Omega A$ is defined as follows. If $A$ is an algebra without filtration or with the trivial filtration, we let $(\Omega A)_{n}$ be the linear span of monomials $\left\langle x_{0}\right\rangle d x_{1} \ldots d x_{m}$ with $m \geq n$. If the algebra $A$ is itself a filtered algebra, we let $(\Omega A)_{n}$ be the linear span of monomials $\left\langle x_{0}\right\rangle d x_{1} \ldots d x_{m}$ with $m \geq n$ or $x_{j} \in A_{n}$ for some $j \in\{0, \ldots, m\}$. The differential, grading, and multiplication are filtered maps. Hence the Fedosov product is filtered and $\mathcal{T} A$ is a filtered algebra. We endow $\Omega^{1}(\mathcal{T} A)=(\mathcal{T} A \oplus \mathbb{C}) \otimes \mathcal{T} A$ and $\Omega^{1}(\mathcal{T} A) /[$, ] with the induced filtrations to turn $X(\mathcal{T} A)$ into a complex of filtered vector spaces. 
Let $C_{\bullet}$ be a complex of filtered vector spaces. The filtered cohomology of $C_{\bullet}$ is defined as the homology of the complex of filtered linear maps $C_{\bullet} \rightarrow \mathbb{C}$. Following Cuntz and Quillen [5], we define $\operatorname{HP}^{*}(A), *=0,1$, as the filtered cohomology of $X(\mathcal{T} A)$. More generally, we define the bivariant periodic cyclic homology $\mathrm{HP}_{*}(A ; B)$ as the homology of the complex of morphisms between the projective systems $\left(X(\mathcal{T} A) / X(\mathcal{T} A)_{n}\right)$ and $\left(X(\mathcal{T} B) / X(\mathcal{T} B)_{n}\right)$ associated to $A$ and $B$.

\section{Bornological vector spaces}

We refer to [8] for the elementary theory of (convex) bornological vector spaces. We will only meet convex bornological vector spaces and therefore omit the qualifier "convex" from our notation. A bornological vector space is a vector space $V$ together with a collection of subsets $\mathfrak{S}(V)$ called the bornology of $V$. The bornology $\mathfrak{S}(V)$ has to satisfy the following conditions. If $S_{1} \subset S_{2}$ and $S_{2} \in \mathfrak{S}(V)$, then $S_{1} \in \mathfrak{S}(V)$. If $S_{1}, S_{2} \in \mathfrak{S}(V)$, then $S_{1} \cup S_{2} \in \mathfrak{S}(V)$. If $S \in \mathfrak{S}(V)$, then $c \cdot S \in \mathfrak{S}(V)$ for all $c \in \mathbb{R}$. We have $\{x\} \in \mathfrak{S}(V)$ for all $x \in V$. If $S \in \mathfrak{S}(V)$, then the disked hull $S^{\circ}$ of $S$ is in $\mathfrak{S}(V)$. The disked hull $S^{\circ}$ of $S$ is defined as the smallest circled convex subset of $V$ containing $S$. We call sets $S \in \mathfrak{S}(V)$ small.

If $V$ is a locally convex topological vector space, then the collection of all bounded subsets of $V$ is a bornology, the bounded bornology on $V$. If $V$ is just a vector space, then there is a finest possible bornology on $V$, namely the collection of all bounded subsets of finite dimensional vector subspaces of $V$.

A linear map $l: V \rightarrow W$ between bornological vector spaces is bounded iff it maps small subsets of $V$ to small subsets of $W$. That is, $l(S) \in \mathfrak{S}(W)$ for all $S \in \mathfrak{S}(V)$. A bornological isomorphism is a bounded linear map with a bounded two-sided inverse. A bilinear map $l: V_{1} \times V_{2} \rightarrow W$ is bounded iff $l\left(S_{1} \times S_{2}\right) \in \mathfrak{S}(W)$ for all $S_{1} \in \mathfrak{S}\left(V_{1}\right), S_{2} \in \mathfrak{S}\left(V_{2}\right)$. A bornological algebra is a bornological vector space $A$ together with a bounded associative multiplication $m: A \times A \rightarrow A$.

There are canonical bornologies on subspaces, quotients, and direct sums of bornological vector spaces [8]. The canonical bornology on the tensor product $V \otimes W$ of two bornological vector spaces $V$ and $W$ is generated by the sets

$$
S_{V} \otimes S_{W}:=\left\{x \otimes y \mid x \in S_{V}, y \in S_{W}\right\}
$$

with $S_{V} \in \mathfrak{S}(V), S_{W} \in \mathfrak{S}(W)$. We write $S \propto T$ iff $S \subset T^{\circ}$. A subset $T \subset V \otimes W$ is small iff $T \subset S_{V} \otimes S_{W}$ for some $S_{V} \in \mathfrak{S}(V), S_{W} \in \mathfrak{S}(W)$. Composition with the natural bilinear map $V \times W \rightarrow V \otimes W$ yields a bijection between bounded bilinear maps $V \times W \rightarrow \sqcup$ and bounded linear maps $V \otimes W \rightarrow \sqcup$. The tensor product bornology is associative and commutative in the usual sense.

Let $C$. be a complex of bornological vector spaces. The bounded cohomology of $C_{\bullet}$ is defined as the homology of the complex of bounded linear maps $C_{\bullet} \rightarrow \mathbb{C}$.

Let $A$ be a filtered bornological algebra and let $V$ be a filtered bornological vector space. The free A-bimodule on $V$ is $A^{+} \otimes V \otimes A^{+}$with the standard bimodule structure and the induced bornology and filtration. It can be characterized by the universal property that composition with the inclusion $V \cong 1 \otimes V \otimes 1 \subset$ 
$A^{+} \otimes V \otimes A^{+}$gives rise to a bijection between filtered bounded linear maps $V \rightarrow W$ and filtered bounded $A$-bimodule homomorphisms $A^{+} \otimes V \otimes A^{+} \rightarrow W$ for any $A$-bimodule $W$. We have $A^{+} \otimes V \otimes A^{+} /[,] \cong A^{+} \otimes V$ as filtered bornological vector spaces. The free left and right $A$-modules on $V$ are $A^{+} \otimes V$ and $V \otimes A^{+}$, respectively. An $A$-(bi)module is projective iff it is a direct summand of a free $A$-(bi)module.

\subsection{Completeness}

For a disk $T$ in a vector space $V$, let $V_{T}$ be the linear span of $T$ in $V$ furnished with the semi-norm whose unit ball is $T$. A disk $T \subset V$ is called completant iff it is the closed unit ball of $V_{T}$ and $V_{T}$ is a Banach space. If each $S \in \mathfrak{S}(V)$ is contained in a completant small disk $T \in \mathfrak{S}(V)$, then $V$ is a complete bornological vector space. A locally convex vector space is called quasi-complete iff each bounded subset is contained in a completant bounded disk iff it is complete as a bornological vector space with respect to the bounded bornology. The following observation illustrates the usefulness of completeness in analysis.

Let $V_{1}, V_{2}$, and $W$ be quasi-complete locally convex vector spaces furnished with the bounded bornology. Then a separately continuous bilinear map $b: V_{1} \times$ $V_{2} \rightarrow W$ is bounded. Otherwise, there would be completant bounded disks $S_{1} \subset$ $V_{1}, S_{2} \subset V_{2}$ such that $b\left(S_{1} \times S_{2}\right)$ is unbounded. The map $\left(V_{1}\right)_{S_{1}} \times\left(V_{2}\right)_{S_{2}} \rightarrow$ $V_{1} \times V_{2} \rightarrow W$ is separately continuous and hence continuous because $\left(V_{1}\right)_{S_{1}}$ and $\left(V_{2}\right)_{S_{2}}$ are Banach spaces. Thus $b\left(S_{1} \times S_{2}\right) \subset W$ is bounded, a contradiction. Therefore, $b$ is bounded.

Hence a quasi-complete locally convex algebra with separately continuous multiplication is a complete bornological algebra with respect to the bounded bornology.

Each bornological vector space $V$ has a completion $V^{\mathrm{c}}$ that is characterized uniquely by a universal property [7]. The completion of a bornological algebra is a complete bornological algebra and has the same entire and periodic cyclic cohomology. Thus we could restrict attention to complete bornological algebras. However, it will be more convenient to avoid completions as much as possible because of the algebraic character of excision. We only need completions to define the bivariant versions of entire and periodic cyclic cohomology.

\section{Entire cyclic cohomology}

Let $A$ be a bornological algebra. For $S \subset A$, let

$$
\langle S\rangle(d S)^{\infty}:=S \cup\left\{\left\langle x_{0}\right\rangle d x_{1} \ldots d x_{n} \mid n \geq 1,\left\langle x_{0}\right\rangle \in S \cup\{1\}, x_{1}, \ldots, x_{n} \in S\right\} .
$$

We will frequently use $\langle S\rangle$ for $S \cup\{1\}$, where 1 behaves like a unit element. For instance, $\langle S\rangle(d S)^{n}:=S(d S)^{n} \cup(d S)^{n}$. We furnish $\Omega A$ with the bornology generated by the sets $\langle S\rangle(d S)^{\infty}$ with $S \in \mathfrak{S}(A)$. Thus $T \in \mathfrak{S}(\Omega A)$ iff $T \subset\langle S\rangle(d S)^{\infty}$ for some $S \in \mathfrak{S}(A)$. We endow $\mathcal{T} A \subset \Omega A$ with the subspace bornology. 
Observe that $\sum_{j=1}^{n}\langle S\rangle(d S)^{n} \Subset\langle 2 S\rangle(d 2 S)^{n}$. Thus we can convert sums into convex combinations by introducing a constant factor. This technique will be useful in many places. It yields that $\langle S\rangle(d S)^{\infty} \cdot\langle S\rangle(d S)^{\infty} \odot\langle T\rangle(d T)^{\infty}$ for $T:=2 S+$ $2 S^{2}$. Hence the multiplication of differential forms is bounded. The differential $d$, the grading operator $\gamma$, and hence the Fedosov product are bounded as well. Consequently, $\mathcal{T} A$ is a bornological algebra. Its $\mathrm{X}$-complex $X(\mathcal{T} A)$ is a complex of bornological vector spaces with respect to the induced bornology on $\Omega^{1}(\mathcal{T} A) /[$, ].

We define the entire cyclic cohomology $\operatorname{HE}^{*}(A)$ of $A$ as the bounded cohomo$\operatorname{logy}$ of $X(\mathcal{T} A)$. In [10], we called this theory analytic cyclic cohomology. Here we stick to Connes's terminology. If $f: A \rightarrow B$ is a bounded homomorphism, then the induced maps $\mathcal{T} f: \mathcal{T} A \rightarrow \mathcal{T} B$ and $X(\mathcal{T} f): X(\mathcal{T} A) \rightarrow X(\mathcal{T} B)$ are bounded as well. Composition with $X(\mathcal{T} f)$ makes $\mathrm{HE}^{*}$ a contravariant functor.

We define the bivariant entire cyclic homology $\operatorname{HE}_{*}(A ; B)$ as the homology of the complex of bounded linear maps $X(\mathcal{T} A)^{\mathrm{c}} \rightarrow X(\mathcal{T} B)^{\mathrm{c}}$. This complex is equal to the complex of bounded linear maps $X(\mathcal{T} A) \rightarrow X(\mathcal{T} B)^{\mathrm{c}}$ by the universal property of completions. We have to complete the target in order to obtain a reasonable homology theory $\mathrm{HE}_{*}(\mathbb{C} ; B)$. The uncompleted complex $X(\mathcal{T} B)$ is acyclic.

If $A$ is a bornological algebra, then we modify the definition of periodic cyclic (co)homology above to take into account the bornology of $A$. Let $\operatorname{HP}^{*}(A)$ be the filtered bounded cohomology of $X(\mathcal{T} A)$, that is, the homology of the complex of filtered bounded linear maps $X(\mathcal{T} A) \rightarrow \mathbb{C}$. In the bivariant theory, we replace the projective system $X\left(\mathcal{T} A /(\mathcal{T} A)_{n}\right)$ by the projective system of completions $X\left(\mathcal{T} A /(\mathcal{T} A)_{n}\right)^{\mathrm{c}}$ and restrict to bounded morphisms of projective systems.

In the following, we will meet many vector spaces that are constructed from $\mathcal{T} A$ and $\Omega A$ by taking quotients, direct sums and tensor products. These spaces are always furnished with the induced bornology and the induced filtration.

\subsection{Comparison to Connes's definition of entire cyclic cohomology}

We denote the standard derivations on $\Omega A$ and $\Omega(\mathcal{T} A)$ by $d$ and $D$, respectively. Let $\sigma_{A}: A \rightarrow \mathcal{T} A$ be the natural linear map $A \cong \Omega^{0} A \subset \Omega^{\text {even }} A=\mathcal{T} A$.

Lemma 1. Let $A$ be a filtered bornological algebra. The map $D \circ \sigma_{A}: A \rightarrow$ $\Omega^{1}(\mathcal{T} A)$ induces a natural isomorphism of filtered bornological $\mathcal{T} A$-bimodules

$$
\begin{aligned}
\mu_{1}:(\mathcal{T} A)^{+} \otimes A \otimes(\mathcal{T} A)^{+} & \rightarrow \Omega^{1}(\mathcal{T} A), \\
& \left\langle x_{0}\right\rangle \otimes a \otimes\left\langle x_{1}\right\rangle \mapsto\left\langle x_{0}\right\rangle \odot\left(D \sigma_{A}(a)\right) \odot\left\langle x_{1}\right\rangle .
\end{aligned}
$$

Thus $\Omega^{1}(\mathcal{T} A)$ is a free $\mathcal{T}$ A-bimodule.

Proof. Cuntz and Quillen show in [4] that $\mu_{1}$ is a vector space isomorphism. It is evident that $\mu_{1}$ is filtered and bounded. We have to check that the inverse map $\mu_{1}^{-1}$ is also filtered and bounded. Since $\mu_{1}^{-1}$ is a bimodule map, it suffices to consider 
its restriction to the subspace $D(\mathcal{T} A)$. Since $D$ is a derivation, we have

$$
\begin{aligned}
\mu_{1}^{-1} D\left(\left\langle a_{0}\right\rangle d a_{1} \ldots d a_{2 n}\right) & =\left(\mu_{1}^{-1} D\left\langle a_{0}\right\rangle\right) \odot d a_{1} \ldots d a_{2 n} \\
& +\sum_{j=0}^{n-1}\left\langle a_{0}\right\rangle d a_{1} \ldots d a_{2 j} \odot \mu_{1}^{-1} D\left(d a_{2 j+1} d a_{2 j+2}\right) \odot d a_{2 j+3} \ldots d a_{2 n} .
\end{aligned}
$$

The big sum can be converted into a convex combination of terms of the form

$$
\left\langle a_{0}\right\rangle d 2 a_{1} \ldots d 2 a_{2 j} \odot \mu_{1}^{-1} D\left(d 2 a_{2 j+1} d 2 a_{2 j+2}\right) \odot d 2 a_{2 j+3} \ldots d 2 a_{2 n} .
$$

The boundedness of $\mu_{1}^{-1}$ follows. It is not hard to show that $\mu_{1}^{-1}$ is filtered.

Hence $\Omega^{1}(\mathcal{T} A) /[,] \cong(\mathcal{T} A)^{+} \otimes A \cong(\mathcal{T} A)^{+} d A=\Omega^{\text {odd }} A$. Thus we obtain a natural isomorphism $X(\mathcal{T} A) \cong \Omega A$ of $\mathbb{Z} / 2$-graded filtered bornological vector spaces.

Let $A$ be a quasi-complete locally convex algebra with separately continuous multiplication. Let $\operatorname{HE}_{C}^{*}(A)$ be its entire cyclic cohomology as defined by Connes [1], [2]. Actually, Connes's original definition only works for unital algebras. Khalkhali has extended it to non-unital algebras in [9]. We rewrite Khalkhali's definition as follows. Endow $A$ with the bounded bornology. Define $[k]=[k+$ $1 / 2]=k$ for all $k \in \mathbb{Z}$. Let $\mathfrak{S}_{n !}$ be the convex bornology on $\Omega A$ generated by the sets

$$
S \cup \bigcup_{n \geq 1}[n / 2] !\langle S\rangle(d S)^{n}
$$

with $S \in \mathfrak{S}(A)$. We furnish the $\mathbb{Z} / 2$-graded vector space $\Omega A$ with the Hodge filtration, the bornology $\mathfrak{S}_{n !}$, and the boundary $B+b$ to obtain a filtered bornological complex $C(A)$. The bounded cohomology of $C(A)$ is equal to $\operatorname{HE}_{C}^{*}(A)$. Indeed, a linear map $l: C(A) \rightarrow \mathbb{C}$ can be described by a family of multi-linear maps $l_{n}: A^{+} \times A^{n} \rightarrow \mathbb{C}, n \geq 0$. The map $l$ is bounded iff for all bounded subsets $S \subset A$, there is a constant $C$ such that $\left|l_{n}\left(\left\langle a_{0}\right\rangle, a_{1}, \ldots, a_{n}\right)\right| \leq C /[n / 2]$ ! for all $n \in \mathbb{N}$, $\left\langle a_{0}\right\rangle \in S \cup\{1\}, a_{1}, \ldots, a_{n} \in S$.

The map $l$ is filtered iff $l_{n}=0$ for all but finitely many $n$. If $l$ is filtered, it is bounded iff $l_{n}$ is bounded for all $n$. Hence the filtered bounded cohomology of $C(A)$ is the "usual" periodic cyclic cohomology of $A$.

Two chain maps between filtered bornological complexes are called chain homotopic iff they differ by the boundary of a filtered bounded linear map. Similarly, if two filtered bornological complexes are called homotopy equivalent, this means that all the four maps involved in the homotopy equivalence are filtered and bounded. Hence if $C_{1}$ and $C_{2}$ are homotopy equivalent, then they have the same bounded and filtered bounded cohomology.

Proposition 1. Let A be a quasi-complete locally convex algebra furnished with the bounded bornology. The filtered bornological complexes $X(\mathcal{T} A)$ and $C(A)$ are naturally homotopy equivalent. Hence the definitions of entire and periodic cyclic cohomology given above agree with the standard definitions. 
Essentially, this is already proved by Cuntz and Quillen in [5]. They construct a homotopy equivalence between the complexes $X(\mathcal{T} A)$ and $(\Omega A, B+b)$. The maps implementing this homotopy equivalence are filtered and bounded. Boundedness is proved by writing down explicit formulas for these maps, see [10] for details. We do not go into these computations here but only observe that the homotopy equivalence involves multiplication by $(-1)^{n} n$ ! in degrees $2 n$ and $2 n+1$. This is where the $[n / 2]$ ! in the definition of the bornology $\mathfrak{S}_{n !}$ enters.

\subsection{Homotopy invariance of the X-complex}

Let $B$ be a filtered bornological algebra. Let $\mathbb{C}[t]$ be the polynomial ring in one variable. We turn $\mathbb{C}[t] \otimes B$ into a filtered bornological algebra as follows. The filtration consists of the subspaces $\mathbb{C}[t] \otimes B_{n}$. For $x \in \mathbb{C}$, let ev $x: \mathbb{C}[t] \otimes B \rightarrow B$ be evaluation at $x$. Let

$$
S \in \mathfrak{S}(\mathbb{C}[t] \otimes B) \Longleftrightarrow\left\{\operatorname{ev}_{x}(f), \operatorname{ev}_{x}(d f / d t) \mid x \in[0,1], f \in S\right\} \in \mathfrak{S}(B) .
$$

Two homomorphisms $f_{0}, f_{1}: A \rightarrow B$ are called polynomially homotopic iff there is a filtered bounded homomorphism $F: A \rightarrow \mathbb{C}[t] \otimes B$ with $\operatorname{ev}_{t} \circ F=f_{t}$ for $t=0,1$.

In general, the chain maps $X\left(f_{0}\right), X\left(f_{1}\right): X(A) \rightarrow X(B)$ induced by polynomially homotopic homomorphisms $f_{0}$ and $f_{1}$ need not be chain homotopic. However, they are chain homotopic if the source $A$ is quasi-free in the sense that the bimodule $\Omega^{1} A$ is projective. Essentially, this is proved already by Cuntz and Quillen [5]. They write down a linear map $h: X(A) \rightarrow X(B)$ with $[\partial, h]=$ $X\left(f_{0}\right)-X\left(f_{1}\right)$. It is straightforward to check that their map $h$ is filtered and bounded. See also [10] for an explanation of the formula for $h$. We will only need homotopy invariance in the special case where $A$ is a tensor algebra (and thus quasi-free by Lemma 1):

Proposition 2. Let $C, B$ be filtered bornological algebras and $A:=\mathcal{T} C$. Let $f_{0}, f_{1}: A \rightarrow B$ be polynomially homotopic homomorphisms. Then the induced chain maps $X\left(f_{0}\right), X\left(f_{1}\right): X(A) \rightarrow X(B)$ are chain homotopic.

\section{The proof of the excision theorem}

Consider an extension $K \rightarrow E \rightarrow Q$ as in Theorem 1. Identify $X(\mathcal{T} E) \cong \Omega E$ and $X(\mathcal{T} Q) \cong \Omega Q$ as above. The section $s$ for $p$ gives rise to a filtered bounded linear section $s_{L}: \Omega Q \rightarrow \Omega E$ for $X(\mathcal{T} p): X(\mathcal{T} E) \rightarrow X(\mathcal{T} Q)$ that is defined by

$$
s_{L}\left(\left\langle q_{0}\right\rangle d q_{1} \ldots d q_{n}\right):=s\left\langle q_{0}\right\rangle d s\left(q_{1}\right) \ldots d s\left(q_{n}\right) .
$$

In addition, we will need the right-handed version

$$
s_{R}\left(d q_{1} \ldots d q_{n} \cdot\left\langle q_{n+1}\right\rangle\right):=d s\left(q_{1}\right) \ldots d s\left(q_{n}\right) \cdot s\left\langle q_{n+1}\right\rangle .
$$


of $s_{L}$. Let

$$
X(\mathcal{T} E: \mathcal{T} Q):=\operatorname{ker}(X(\mathcal{T} p): X(\mathcal{T} E) \rightarrow X(\mathcal{T} Q))
$$

The chain map $X(\mathcal{T} i): X(\mathcal{T} K) \rightarrow X(\mathcal{T} E)$ satisfies $X(\mathcal{T} p) \circ X(\mathcal{T} i)=0$, so that we can view it as a chain map $\varrho: X(\mathcal{T} K) \rightarrow X(\mathcal{T} E: \mathcal{T} Q)$. We will show that $\varrho$ is a homotopy equivalence of filtered bornological complexes. This implies excision in (bivariant) entire and periodic cyclic cohomology. We have an extension

$$
X(\mathcal{T} E: \mathcal{T} Q) \stackrel{\subset}{\longrightarrow} X(\mathcal{T} E) \stackrel{X(\mathcal{T} p)}{\longrightarrow} X(\mathcal{T} Q)
$$

of filtered bornological complexes with a filtered bounded linear section. Let $C$ be another (filtered) bornological complex. Let $\operatorname{Lin}(V ; W)$ be the space of (filtered) bounded linear maps $V \rightarrow W$. Since the extension (2) splits, it remains an extension of complexes of vector spaces after applying the functors $\operatorname{Lin}\left(C ; \sqcup^{\mathrm{c}}\right)$ or $\operatorname{Lin}\left(\sqcup ; C^{\mathrm{c}}\right)$. In the associated exact homology sequence, we can replace $X(\mathcal{T} E$ : $\mathcal{T} Q)$ by $X(\mathcal{T} K)$ if $\varrho$ is a homotopy equivalence. This yields the desired six term exact sequences.

Let $\mathcal{L}$ be the left ideal in $\mathcal{T} E$ generated by $K \subset E \subset \mathcal{T} E$. Alternatively, we can define $\mathcal{L}$ as the linear span of the monomials $\left\langle x_{0}\right\rangle d x_{1} \ldots d x_{2 n}$ with $x_{2 n} \in K$. Our ultimate goals are to show that $X(\mathcal{L}) \sim X(\mathcal{T} E: \mathcal{T} Q)$ and $X(\mathcal{T} K) \sim X(\mathcal{L})$, where $\sim$ denotes homotopy equivalence of filtered bornological complexes.

Some conventions: We write $x, x_{j}, j \in \mathbb{N}$, for elements of $E ; y, y_{j}$ for elements of $\mathcal{T} E ; l, l_{j}$ for elements of $\mathcal{L} ; q, q_{j}$ for elements of $Q$ and $\mathcal{T} Q ; k$ for elements of $K ;\langle x\rangle$ for elements of $E^{+}$, and similarly $\langle y\rangle,\langle l\rangle,\langle q\rangle$. We write $d$ and $D$ for the standard derivations of $\Omega E$ and $\Omega(\mathcal{T} E)$. We declare $d 1=0$ and $D 1=0$. An expression $d x_{1} \ldots d x_{2 n}$ or $D y_{1} \ldots D y_{2 n}$ with $n=0$ is 1 . We will frequently use the grading \# on $(\mathcal{T} E)^{+} \supset \mathcal{L}^{+}$defined by \#1 $=0$ and \#y=n iff $y \in \Omega^{2 n} E$.

\subsection{A free resolution}

Let $\psi: X(\mathcal{L}) \rightarrow X(\mathcal{T} E: \mathcal{T} Q)$ be the chain map induced by the inclusion $\mathcal{L} \subset$ $\mathcal{T} E$. Let $C_{\bullet}$. be the contractible complex $C_{0}=C_{1}=\mathcal{T} Q \otimes \mathcal{L}$ with boundary id: $C_{1} \rightarrow C_{0}$. We define a chain map $\psi^{\prime}: C_{\bullet} \rightarrow X(\mathcal{T} E: \mathcal{T} Q)$ by $C_{0} \ni q \otimes l \mapsto$ $\left[l, s_{L}(q)\right] \in \mathcal{T} E$ and $C_{1} \ni q \otimes l \mapsto l D s_{L}(q) \bmod [,] \in X_{1}(\mathcal{T} E)$.

Lemma 2. The map $\left(\psi, \psi^{\prime}\right): X(\mathcal{L}) \oplus C_{\bullet} \rightarrow X(\mathcal{T} E: \mathcal{T} Q)$ is a filtered bornological isomorphism. Thus $\psi: X(\mathcal{L}) \rightarrow X(\mathcal{T} E: \mathcal{T} Q)$ is a homotopy equivalence.

The map $\left(\psi, \psi^{\prime}\right): X_{\beta}(\mathcal{L}) \oplus C_{\bullet} \rightarrow X_{\beta}(\mathcal{T} E: \mathcal{T} Q)$ is a filtered bornological isomorphism. Thus $\psi: X_{\beta}(\mathcal{L}) \rightarrow X_{\beta}(\mathcal{T} E: \mathcal{T} Q)$ is a homotopy equivalence.

Recall that the $X$-complex and the $X_{\beta}$-complex are equal as filtered bornological vector spaces. Hence the two paragraphs of Lemma 2 are equivalent. We will prove the second paragraph by homological algebra. 
Let $A$ be a filtered bornological algebra. Consider the following extension

$$
\begin{aligned}
\Omega^{1} A \stackrel{\alpha_{1}}{\longrightarrow} A^{+} \otimes A^{+} \stackrel{\alpha_{0}}{\longrightarrow} A^{+}, \\
\alpha_{1}(\langle x\rangle D y):=\langle x\rangle \odot y \otimes 1-\langle x\rangle \otimes y, \\
\alpha_{0}(\langle x\rangle \otimes\langle y\rangle):=\langle x\rangle \cdot\langle y\rangle .
\end{aligned}
$$

A contracting homotopy $h_{0}: A^{+} \rightarrow A^{+} \otimes A^{+}, h_{1}: A^{+} \otimes A^{+} \rightarrow \Omega^{1} A$ is defined by $h_{0}(\langle x\rangle):=1 \otimes\langle x\rangle$ and $h_{1}(\langle x\rangle \otimes\langle y\rangle):=(D\langle x\rangle)\langle y\rangle$. It is straightforward to verify that the maps $\alpha_{\bullet}$ are $A$-bimodule homomorphisms and that $\alpha_{\bullet}^{2}=0$ and $\alpha_{\bullet} h_{\bullet}+h_{\bullet} \alpha_{\bullet}=1$. We write $B_{\bullet}^{A}$ for the complex $\Omega^{1} A \stackrel{\alpha}{\rightarrow} A^{+} \otimes A^{+}$. Let $\mathbb{C}[0]$ be the complex with $\mathbb{C}$ in degree zero and 0 in all other degrees. We have

$$
B_{\bullet}^{A} /[,]:=B_{\bullet}^{A} /\left[B_{\bullet}^{A}, A\right] \cong X_{\beta}(A) \oplus \mathbb{C}[0] .
$$

We define another $\mathcal{L}$-bimodule resolution $P_{1} \rightarrow P_{0} \rightarrow \mathcal{L}^{+}$of $\mathcal{L}^{+}$by

$$
\begin{aligned}
& P_{0}:=\mathcal{L}^{+} \otimes \mathcal{L}^{+}+(\mathcal{T} E)^{+} \otimes \mathcal{L} \subset(\mathcal{T} E)^{+} \otimes(\mathcal{T} E)^{+}=B_{0}^{\mathcal{T} E} \\
& P_{1}:=(\mathcal{T} E)^{+} D \mathcal{L} \subset \Omega^{1}(\mathcal{T} E)=B_{1}^{\mathcal{T} E}
\end{aligned}
$$

The subspaces $P_{0} \subset B_{0}^{\mathcal{T} E}$ and $P_{1} \subset B_{1}^{\mathcal{T} E}$ are sub- $\mathcal{L}$-bimodules. We have

$$
\alpha_{1}\left(P_{1}\right) \subset P_{0}, \quad \alpha_{0}\left(P_{0}\right) \subset \mathcal{L}^{+}, \quad h_{0}\left(\mathcal{L}^{+}\right) \subset P_{0}, \quad h_{1}\left(P_{0}\right) \subset P_{1},
$$

because $\mathcal{L}$ is a left ideal in $(\mathcal{T} E)^{+}$. Thus $\left(P_{\bullet}, \alpha_{\bullet}\right)$ is an $\mathcal{L}$-bimodule resolution of $\mathcal{L}^{+}$.

We are going to show that $P_{0}$ and $P_{1}$ are free $\mathcal{L}$-bimodules in order to compute their commutator quotients: $P_{\bullet} /[,] \cong X_{\beta}(\mathcal{T} E: \mathcal{T} Q) \oplus \mathbb{C}[0]$. Furthermore, we compare the $\mathcal{L}$-bimodule resolutions $P_{\bullet}$ and $B_{\bullet}^{\mathcal{L}}$ and obtain $P_{\bullet} \cong B_{\bullet}^{\mathcal{L}} \oplus\left(\mathcal{L}^{+} \otimes C_{\bullet}\right)$. These facts together imply Lemma 2 . First, we need some preparations.

Lemma 3. Let $A$ be a filtered bornological algebra. The natural maps

$$
\begin{array}{ll}
m_{L}:(\mathcal{T} A)^{+} \otimes A \rightarrow \mathcal{T} A, & \langle y\rangle \otimes x \mapsto\langle y\rangle \odot \sigma_{A}(x), \\
m_{R}: A \otimes(\mathcal{T} A)^{+} \rightarrow \mathcal{T} A, & x \otimes\langle y\rangle \mapsto \sigma_{A}(x) \odot\langle y\rangle
\end{array}
$$

are filtered bornological isomorphisms.

Proof. We only prove that $m_{L}$ is a filtered bornological isomorphism. The proof for $m_{R}$ is similar. Let $m_{L}^{-1}(x):=1 \otimes x$ for all $x \in A$. Monomials of higher degree are of the form $\langle y\rangle d x_{1} d x_{2}$ with $\langle y\rangle \in(\mathcal{T} A)^{+}, x_{1}, x_{2} \in A$. Let

$$
m_{L}^{-1}\left(\langle y\rangle d x_{1} d x_{2}\right):=\langle y\rangle \otimes\left(x_{1} \cdot x_{2}\right)-\left(\langle y\rangle \odot x_{1}\right) \otimes x_{2} .
$$

It is straightforward to verify that $m_{L} \circ m_{L}^{-1}=\mathrm{id}$ and $m_{L}^{-1} \circ m_{L}=\mathrm{id}$. Moreover, $m_{L}^{-1}$ is filtered and bounded. Thus $m_{L}$ is a filtered bornological isomorphism. 
If we let $A=E$, then $m_{L}$ maps the direct summand $(\mathcal{T} E)^{+} \otimes K \subset(\mathcal{T} E)^{+} \otimes E$ onto the smallest left ideal that contains $K$, that is, $\mathcal{L}$. Hence we obtain a natural filtered bornological isomorphism

$$
\mu_{3}:(\mathcal{T} E)^{+} \otimes K \rightarrow \mathcal{L}, \quad\langle y\rangle \otimes k \mapsto\langle y\rangle \odot k .
$$

Let $\mathcal{I}$ be the kernel of the homomorphism $\mathcal{T} p: \mathcal{T} E \rightarrow \mathcal{T} Q$.

Lemma 4. The following two linear maps are filtered bornological isomorphisms:

$$
\begin{array}{ll}
\mu_{4}:(\mathcal{T} E)^{+} \otimes K \otimes(\mathcal{T} Q)^{+} \rightarrow \mathcal{I}, & \langle y\rangle \otimes k \otimes\langle q\rangle \mapsto\langle y\rangle \odot k \odot s_{L}\langle q\rangle, \\
\mu_{5}:(\mathcal{T} Q)^{+} \otimes K \otimes(\mathcal{T} E)^{+} \rightarrow \mathcal{I}, & \langle q\rangle \otimes k \otimes\langle y\rangle \mapsto s_{R}\langle q\rangle \odot k \odot\langle y\rangle .
\end{array}
$$

The restriction of $\mu_{5}$ to $(\mathcal{T} Q)^{+} \otimes K \otimes \mathcal{L}^{+}$is a filtered bornological isomorphism onto $\mathcal{L}$. Thus $\mathcal{L}$ is free as a right $\mathcal{L}$-module.

Proof. We prove that (5) is an isomorphism by writing down the inverse map and checking that it is filtered and bounded. The proof for (4) is analogous.

We call $d x_{1} \ldots d x_{2 n}\left\langle x_{2 n+1}\right\rangle$ a standard monomial iff $x_{j} \in K \cup s(Q)$ for $j=$ $0, \ldots, 2 n$ and $\left\langle x_{2 n+1}\right\rangle \in\{1\} \cup K \cup s(Q)$. Elements of $\mathcal{I}$ are linear combinations of standard monomials with at least one entry in $K$. We are going to write down a $\mu_{5}$-preimage for such a standard monomial $d x_{1} \ldots d x_{2 n}\left\langle x_{2 n+1}\right\rangle \in \mathcal{I}$. We pick the first $j$ with $x_{j} \in K$, so that $x_{i}=s\left(q_{i}\right)$ for $i<j$ with $q_{i}=p\left(x_{i}\right)$. We distinguish three cases: $j$ is even; $j$ is odd and $j \leq 2 n-1$; or $j=2 n+1$. If $j$ is even, then

$$
\begin{aligned}
& \mu_{5}^{-1}\left(d x_{1} \ldots d x_{2 n}\left\langle x_{2 n+1}\right\rangle\right) \\
& =\mu_{5}^{-1}\left(d s q_{1} \ldots d s q_{j-2} \odot\left(s q_{j-1} \cdot x_{j}-s q_{j-1} \odot x_{j}\right) \odot d x_{j+1} \ldots d x_{2 n}\left\langle x_{2 n+1}\right\rangle\right) \\
& \quad=d q_{1} \ldots d q_{j-2} \otimes s q_{j-1} x_{j} \otimes d x_{j+1} \ldots d x_{2 n}\left\langle x_{2 n+1}\right\rangle \\
& \quad-d q_{1} \ldots d q_{j-2} \cdot q_{j-1} \otimes x_{j} \otimes d x_{j+1} \ldots d x_{2 n}\left\langle x_{2 n+1}\right\rangle .
\end{aligned}
$$

If $j$ is odd and $j \leq 2 n-1$, then we obtain similarly

$$
\begin{array}{r}
\mu_{5}^{-1}\left(d x_{1} \ldots d x_{2 n}\left\langle x_{2 n+1}\right\rangle\right)=d q_{1} \ldots d q_{j-1} \otimes x_{j} x_{j+1} \otimes d x_{j+2} \ldots d x_{2 n}\left\langle x_{2 n+1}\right\rangle \\
-d q_{1} \ldots d q_{j-1} \otimes x_{j} \otimes x_{j+1} \odot d x_{j+2} \ldots d x_{2 n}\left\langle x_{2 n+1}\right\rangle .
\end{array}
$$

Finally, if $j=2 n+1$, then $\left\langle x_{2 n+1}\right\rangle=x_{2 n+1} \in K$ and

$$
\mu_{5}^{-1}\left(d x_{1} \ldots d x_{2 n}\left\langle x_{2 n+1}\right\rangle\right)=d q_{1} \ldots d q_{2 n} \otimes x_{2 n+1} \otimes 1
$$

These formulas yield a linear map $\mu_{5}^{-1}: \mathcal{I} \rightarrow(\mathcal{T} Q)^{+} \otimes K \otimes(\mathcal{T} E)^{+}$such that $\mu_{5} \circ \mu_{5}^{-1}=\mathrm{id}$. It is left to the reader to check $\mu_{5}^{-1} \circ \mu_{5}=$ id. Hence $\mu_{5}$ is an isomorphism with inverse $\mu_{5}^{-1}$. It is straightforward to check that $\mu_{5}$ maps $(\mathcal{T} Q)^{+} \otimes K \otimes \mathcal{L}^{+}$into $\mathcal{L}$ and that $\mu_{5}^{-1}$ maps $\mathcal{L}$ into $(\mathcal{T} Q)^{+} \otimes K \otimes \mathcal{L}^{+}$. Hence the restriction of $\mu_{5}$ to $(\mathcal{T} Q)^{+} \otimes K \otimes \mathcal{L}^{+}$is an isomorphism onto $\mathcal{L}$.

It remains to show that $\mu_{5}^{-1}$ is filtered and bounded. The following estimates will be needed later. The above formulas for $\mu_{5}^{-1}$ show that $\mu_{5}^{-1}(y)$ is a sum of 
terms $\left\langle y_{0}\right\rangle \otimes k \otimes\left\langle y_{1}\right\rangle$ with $\#\left\langle y_{0}\right\rangle+\#\left\langle y_{1}\right\rangle \geq \# y-1$. That is, $\mu_{5}^{-1}$ decreases \# by at most 1 . Thus $\mu_{5}^{-1}$ is filtering.

For $S \in \mathfrak{S}(E)$, let

$$
(d S)^{\mathrm{even}}\langle S\rangle:=\bigcup_{n \geq 0}(d S)^{2 n}\langle S\rangle \subset(\mathcal{T} E)^{+} .
$$

Thus $1 \in(d S)^{\text {even }}\langle S\rangle$. If $T \subset \mathcal{I}$ is small, then $T \subseteq(d S)^{\text {even }}\langle S\rangle \cap \mathcal{I}$ for some $S \in \mathfrak{S}(E)$. We have $S \Subset S_{E}:=S_{K} \cup s\left(S_{Q}\right)$ for suitable $S_{K} \in \mathfrak{S}(K), S_{Q} \in \mathfrak{S}(Q)$ and therefore $T \Subset\left(d S_{E}\right)^{\text {even }}\left\langle S_{E}\right\rangle \cap \mathcal{I}$. The above formulas for $\mu_{5}^{-1}$ show that

$$
\begin{array}{r}
\mu_{5}^{-1}\left(\left(d S_{E}\right)^{\text {even }}\left\langle S_{E}\right\rangle \cap \mathcal{I}\right) \\
\quad \subseteq\left(d S_{Q}\right)^{\text {even }}\left\langle S_{Q}\right\rangle \otimes\left\langle S_{E}\right\rangle \cdot S_{K} \cdot\left\langle S_{E}\right\rangle \otimes 2\left\langle S_{E}\right\rangle \odot\left(d S_{E}\right)^{\text {even }}\left\langle S_{E}\right\rangle .
\end{array}
$$

Since the right hand side in (6) is small, $\mu_{5}^{-1}$ is bounded.

Using (3) and (4), we obtain a filtered bornological isomorphism $\mathcal{L} \otimes(\mathcal{T} Q)^{+}$ $\cong \mathcal{I}$. Since $(\mathcal{T} E)^{+} \cong \mathcal{I} \oplus s_{L}(\mathcal{T} Q)^{+}$, the map

$$
\mu_{7}: \mathcal{L}^{+} \otimes(\mathcal{T} Q)^{+} \rightarrow(\mathcal{T} E)^{+}, \quad\langle l\rangle \otimes\langle q\rangle \mapsto\langle l\rangle \odot s_{L}\langle q\rangle,
$$

is a filtered bornological isomorphism. By construction, $\mu_{7}$ is a left $\mathcal{L}$-module map. Thus $(\mathcal{T} E)^{+}$is a free left $\mathcal{L}$-module.

Finally, we can prove that $P_{0}:=\mathcal{L}^{+} \otimes \mathcal{L}^{+}+(\mathcal{T} E)^{+} \otimes \mathcal{L}$ and $P_{1}:=(\mathcal{T} E)^{+} D \mathcal{L}$ are free $\mathcal{L}$-bimodules. Equation (7) yields an $\mathcal{L}$-bimodule isomorphism

$$
P_{0} \cong \mathcal{L}^{+} \otimes \mathcal{L}^{+}+\mathcal{L}^{+} \otimes(\mathcal{T} Q)^{+} \otimes \mathcal{L} \cong\left(\mathcal{L}^{+} \otimes \mathcal{L}^{+}\right) \oplus\left(\mathcal{L}^{+} \otimes \mathcal{T} Q \otimes \mathcal{L}\right) .
$$

Since $\mathcal{L}$ is a free right $\mathcal{L}$-module, it follows that $P_{0}$ is a free $\mathcal{L}$-bimodule.

We claim that $P_{1} \subset \Omega^{1}(\mathcal{T} E)$ is equal to $\Omega^{1}(\mathcal{T} E) \odot K+(\mathcal{T} E)^{+} D K \subset$ $\Omega^{1}(\mathcal{T} E)$. Using $\left\langle y_{0}\right\rangle D\left(\left\langle y_{1}\right\rangle \odot k\right)=\left(\left\langle y_{0}\right\rangle D\left\langle y_{1}\right\rangle\right) \odot k+\left\langle y_{0}\right\rangle \odot\left\langle y_{1}\right\rangle D k$ and (3), we conclude that $(\mathcal{T} E)^{+} D \mathcal{L}$ and $\Omega^{1}(\mathcal{T} E) \odot K$ agree modulo $(\mathcal{T} E)^{+} D K$. Since $(\mathcal{T} E)^{+} D K \subset(\mathcal{T} E)^{+} D \mathcal{L}$, we get $P_{1}=\Omega^{1}(\mathcal{T} E) \odot K+(\mathcal{T} E)^{+} D K$. The isomorphism

$$
\mu_{1}^{-1}: \Omega^{1}(\mathcal{T} E) \rightarrow(\mathcal{T} E)^{+} \otimes E \otimes(\mathcal{T} E)^{+}
$$

of Lemma 1 maps $(\mathcal{T} E)^{+} D K$ onto $(\mathcal{T} E)^{+} \otimes K \otimes 1$ and maps $\Omega^{1}(\mathcal{T} E) \odot K$ onto $(\mathcal{T} E)^{+} \otimes E \otimes(\mathcal{T} E)^{+} \odot K=(\mathcal{T} E)^{+} \otimes K \otimes \mathcal{L}+(\mathcal{T} E)^{+} \otimes s(Q) \otimes \mathcal{L}$ because $\mu_{1}^{-1}$ is a $\mathcal{T} E$-bimodule homomorphism. Thus $\mu_{1}^{-1}$ restricts to an isomorphism

$$
P_{1} \cong(\mathcal{T} E)^{+} \otimes K \otimes \mathcal{L}^{+} \oplus(\mathcal{T} E)^{+} \otimes Q \otimes \mathcal{L}
$$

of filtered bornological $\mathcal{L}$-bimodules. It follows that $P_{1}$ is a free $\mathcal{L}$-bimodule.

The inclusion $P_{\bullet} \subset B_{\bullet}^{\mathcal{T} E}$ induces a chain map

$$
\phi_{\bullet}: P_{\bullet} /\left[P_{\bullet}, \mathcal{L}\right] \rightarrow B_{\bullet}^{\mathcal{T} E} /\left[B_{\bullet}^{\mathcal{T} E}, \mathcal{T} E\right] \cong X_{\beta}(\mathcal{T} E) \oplus \mathbb{C}[0] .
$$

We claim that $\phi_{\bullet}$ is a filtered bornological isomorphism onto $X_{\beta}(\mathcal{T} E: \mathcal{T} Q) \oplus \mathbb{C}[0]$. This is verified by computing the left hand side $P_{\bullet} /[$, ]. We have

$$
P_{0} /[,] \cong \mathcal{L}^{+} \oplus \mathcal{L} \otimes \mathcal{T} Q \cong \mathbb{C} \oplus \mathcal{L} \otimes(\mathcal{T} Q)^{+} \cong \mathbb{C} \oplus \mathcal{I}=\mathbb{C} \oplus X_{\beta}(\mathcal{T} E: \mathcal{T} Q)_{0}
$$


by (4) and (3). The isomorphism $X_{\beta}(\mathcal{T} E)_{1} \rightarrow \Omega^{\text {odd }} E$ maps $X_{\beta}(\mathcal{T} E: \mathcal{T} Q)_{1}$ onto $\mathcal{I} d s(Q) \oplus(\mathcal{T} E)^{+} d K$. Using (4) and (7), we compute

$$
\begin{aligned}
& P_{1} /[,] \cong \mathcal{L}^{+} \otimes(\mathcal{T} Q)^{+} \otimes K \oplus \mathcal{L} \otimes(\mathcal{T} Q)^{+} \otimes Q \cong(\mathcal{T} E)^{+} \otimes K \oplus \mathcal{I} \otimes Q \\
& \cong(\mathcal{T} E)^{+} d K \oplus \mathcal{I} d s(Q)=X_{\beta}(\mathcal{T} E: \mathcal{T} Q)_{1}
\end{aligned}
$$

It is not hard to check that these isomorphisms $P_{0} /[,] \rightarrow X_{\beta}(\mathcal{T} E: \mathcal{T} Q)_{0} \oplus \mathbb{C}$ and $P_{1} /[,] \rightarrow X_{\beta}(\mathcal{T} E: \mathcal{T} Q)_{1}$ are equal to $\phi_{0}$ and $\phi_{1}$, respectively.

We construct an isomorphism $P_{\bullet} \cong B_{\bullet}^{\mathcal{L}} \oplus\left(\mathcal{L}^{+} \otimes C_{\bullet}\right)$ of complexes of $\mathcal{L}$-bimodules. Recall that $C_{0}=C_{1}=\mathcal{T} Q \otimes \mathcal{L}$. There is a natural inclusion $B_{\bullet}^{\mathcal{L}} \subset P_{\bullet}$ because $\mathcal{L}^{+} \otimes \mathcal{L}^{+} \subset P_{0}$ and $\Omega^{1} \mathcal{L} \subset P_{1}$. The inclusion $\mathcal{L}^{+} \otimes C_{\bullet} \rightarrow P_{\bullet}$ is defined by

$$
\begin{array}{ll}
\mathcal{L}^{+} \otimes C_{1} \rightarrow P_{1}, & \left\langle l_{0}\right\rangle \otimes q \otimes l_{1} \mapsto\left\langle l_{0}\right\rangle\left(D s_{L}(q)\right) l_{1}, \\
\mathcal{L}^{+} \otimes C_{0} \rightarrow P_{0}, & \left\langle l_{0}\right\rangle \otimes q \otimes l_{1} \mapsto\left\langle l_{0}\right\rangle \odot s_{L}(q) \otimes l_{1}-\left\langle l_{0}\right\rangle \otimes s_{L}(q) \odot l_{1} .
\end{array}
$$

The induced map $B_{0}^{\mathcal{L}} \oplus\left(\mathcal{L}^{+} \otimes C_{0}\right) \rightarrow P_{0}$ is a filtered bornological isomorphism by (8). Hence we obtain a filtered bornological isomorphism

$$
B_{1}^{\mathcal{L}} \oplus \mathcal{L}^{+} \otimes C_{1} \cong \operatorname{ker}\left(B_{0}^{\mathcal{L}} \oplus \mathcal{L}^{+} \otimes C_{0} \rightarrow \mathcal{L}^{+}\right) \cong \operatorname{ker}\left(P_{0} \rightarrow \mathcal{L}^{+}\right) \cong P_{1}
$$

Thus $B_{\bullet}^{\mathcal{L}} \oplus\left(\mathcal{L}^{+} \otimes C_{\bullet}\right) \cong P_{\bullet}$ as desired. Taking commutator quotients, we get

$$
X_{\beta}(\mathcal{L}) \oplus \mathbb{C}[0] \oplus C_{\bullet} \cong P_{\bullet} /[,] .
$$

The composition of the above isomorphisms

$$
X_{\beta}(\mathcal{L}) \oplus C_{\bullet} \oplus \mathbb{C}[0] \rightarrow P_{\bullet} /[,] \rightarrow X_{\beta}(\mathcal{T} E: \mathcal{T} Q) \oplus \mathbb{C}[0]
$$

is equal to the map $\left(\psi, \psi^{\prime}\right) \oplus \mathrm{id}_{\mathbb{C}}$, with $\psi$ and $\psi^{\prime}$ as in Lemma 2. Consequently, $\left(\psi, \psi^{\prime}\right)$ is a filtered bornological isomorphism. The proof of Lemma 2 is finished.

Moreover, we obtain that $\Omega^{1} \mathcal{L}=B_{1}^{\mathcal{L}}$ is projective as a direct summand of the free $\mathcal{L}$-bimodule $P_{1}$. Thus $\mathcal{L}$ is quasi-free. We will not use this observation.

\subsection{A left $\mathcal{T}$ E-action on $\mathcal{T} \mathcal{L}$}

We construct a homomorphism $v: \mathcal{L} \rightarrow \mathcal{T} \mathcal{L}$ that will be used to prove that $X(\mathcal{T} K) \sim X(\mathcal{L})$

First we establish some conventions. For any algebra $A$, let $\tau_{A}: \mathcal{T} A \rightarrow A$ be the natural projection with $\left.\tau_{A}\right|_{\Omega^{0} A}=$ id and $\left.\tau_{A}\right|_{\Omega^{2 n} A}=0$ for all $n \neq 0$. We always consider $K \subset \mathcal{L} \subset \mathcal{T} \mathcal{L}$ via the natural linear maps $\sigma_{E}: E \rightarrow \mathcal{T} E$ and $\sigma_{\mathcal{L}}: \mathcal{L} \rightarrow \mathcal{T} \mathcal{L}$. We write $\odot$ for the Fedosov product in $\mathcal{T} \mathcal{L}$ and denote elements of $\mathcal{T} \mathcal{L}$ by $z, z_{j}$.

Let $G:=s_{R}(\mathcal{T} Q)^{+} \odot K \subset \mathcal{L}$. Let $\alpha: \mathcal{I} \rightarrow G D(\mathcal{T} E) \subset \Omega^{1}(\mathcal{T} E)$ be the composition of the isomorphism $\mu_{5}^{-1}: \mathcal{I} \rightarrow(\mathcal{T} Q)^{+} \otimes K \otimes(\mathcal{T} E)^{+}$with the linear map sending $\langle q\rangle \otimes k \otimes\langle y\rangle$ to $s_{R}\langle q\rangle \odot k D\langle y\rangle$. Thus $\left.\alpha\right|_{G}=0$ and $\alpha(g \odot y):=g D y$ for all $g \in G, y \in \mathcal{T} E$. Restricting $\alpha$ to $\mathcal{L}$, we obtain a map $\alpha: \mathcal{L} \rightarrow \mathcal{L} D \mathcal{L} \subset \Omega^{1} \mathcal{L}$. 
Define a filtered bounded bilinear map $E \times \mathcal{T} \mathcal{L} \rightarrow \mathcal{T} \mathcal{L},(e, z) \mapsto e \triangleright z$, by

$$
x \triangleright(l \odot\langle z\rangle):=(x \odot l) \odot\langle z\rangle-D \alpha(x \odot l) \odot\langle z\rangle
$$

This is well-defined because $\mathcal{T} \mathcal{L} \cong \mathcal{L} \otimes(\mathcal{T} \mathcal{L})^{+}$by Lemma 3 .

By construction, $x \triangleright\left(z_{0} \odot z_{1}\right)=\left(x \triangleright z_{0}\right) \odot z_{1}$. We have $\alpha\left(g \odot\left\langle y_{0}\right\rangle \odot\left\langle y_{1}\right\rangle\right)=$ $g D\left(\left\langle y_{0}\right\rangle \odot\left\langle y_{1}\right\rangle\right)=\alpha\left(g \odot\left\langle y_{0}\right\rangle\right) \odot\left\langle y_{1}\right\rangle+g \odot\left\langle y_{0}\right\rangle D\left\langle y_{1}\right\rangle$. Thus

$$
\alpha\left(y_{0} \odot\left\langle y_{1}\right\rangle\right)=\alpha\left(y_{0}\right) \odot\left\langle y_{1}\right\rangle+y_{0} D\left\langle y_{1}\right\rangle \quad \forall y_{0} \in \mathcal{I},\left\langle y_{1}\right\rangle \in \mathcal{T} E .
$$

Using also that $D$ is a graded derivation, we get

$$
\begin{array}{r}
x \triangleright D l_{1} D l_{2}=x \odot l_{1} \odot l_{2}-D \alpha\left(x \odot l_{1} \odot l_{2}\right)-\left(x \odot l_{1}\right) \odot l_{2}+D \alpha\left(x \odot l_{1}\right) \odot l_{2} \\
=-D\left(\alpha\left(x \odot l_{1}\right) \odot l_{2}\right)+D \alpha\left(x \odot l_{1}\right) \odot l_{2}=\alpha\left(x \odot l_{1}\right) D l_{2} .
\end{array}
$$

Hence we can alternatively define $\triangleright$ by

$$
\begin{aligned}
x \triangleright\left\langle l_{0}\right\rangle D l_{1} \ldots D l_{2 n} & =x \odot\left\langle l_{0}\right\rangle D l_{1} \ldots D l_{2 n}-D \alpha\left(x \odot\left\langle l_{0}\right\rangle\right) D l_{1} \ldots D l_{2 n}, \\
x & \triangleright D l_{1} \ldots D l_{2 n}=\alpha\left(x \odot l_{1}\right) D l_{2} \ldots D l_{2 n} .
\end{aligned}
$$

We view $\triangleright$ as a linear map $E \rightarrow \operatorname{Lin}(\mathcal{T} \mathcal{L} ; \mathcal{T} \mathcal{L})$ sending $x \in E$ to the endomorphism $z \mapsto x \triangleright z$ of $\mathcal{T} \mathcal{L}$. We can extend this linear map uniquely to a unital homomorphism $\triangleright:(\mathcal{T} E)^{+} \rightarrow \operatorname{Lin}(\mathcal{T} \mathcal{L} ; \mathcal{T} \mathcal{L})$. Let $v(\langle y\rangle \odot k):=\langle y\rangle \triangleright k$ for all $\langle y\rangle \in(\mathcal{T} E)^{+}, k \in K$. That is, evaluate the endomorphism $\triangleright\langle y\rangle$ on $k \in K \subset \mathcal{T} \mathcal{L}$. This well-defines a linear map $v: \mathcal{L} \rightarrow \mathcal{T} \mathcal{L}$ because $\mathcal{L} \cong(\mathcal{T} E)^{+} \otimes K$.

Lemma 5. The map $v$ is a filtered bounded homomorphism satisfying $\tau_{\mathcal{L}} \circ v=\mathrm{id}{ }_{\mathcal{L}}$. In addition, $\left.v\right|_{K}$ is the standard inclusion $K \leq \mathcal{T} \mathcal{L}$.

Proof. Since $K \subset G$, we have $\alpha(k \odot l)=k D l$ and hence $k \triangleright z=k \odot z$ for all $k \in K, z \in \mathcal{T} \mathcal{L}$. We have $\langle y\rangle \triangleright\left(z_{0} \odot z_{1}\right)=\left(\langle y\rangle \triangleright z_{0}\right) \odot z_{1}$ for all $\langle y\rangle \in(\mathcal{T} E)^{+}$, $z_{0}, z_{1} \in \mathcal{T} \mathcal{L}$ because $E$ generates $\mathcal{T} E$. Therefore,

$$
\begin{array}{r}
v\left(\left\langle y_{0}\right\rangle \odot k_{0} \odot\left\langle y_{1}\right\rangle \odot k_{1}\right)=\left\langle y_{0}\right\rangle \triangleright k_{0} \triangleright\left\langle y_{1}\right\rangle \triangleright k_{1}=\left\langle y_{0}\right\rangle \triangleright\left(k_{0} \odot\left\langle y_{1}\right\rangle \triangleright k_{1}\right) \\
=\left(\left\langle y_{0}\right\rangle \triangleright k_{0}\right) \odot\left(\left\langle y_{1}\right\rangle \triangleright k_{1}\right)=v\left(\left\langle y_{0}\right\rangle \odot k_{0}\right) \odot v\left(\left\langle y_{1}\right\rangle \odot k_{1}\right),
\end{array}
$$

so that $v$ is a homomorphism.

Equations (9) and (10) imply $\tau_{\mathcal{L}}(x \triangleright z)=x \odot \tau_{\mathcal{L}}(z)$ for all $x \in E, z \in \mathcal{T} \mathcal{L}$. Consequently, $\tau_{\mathcal{L}}(\langle y\rangle \triangleright z)=\langle y\rangle \odot \tau_{\mathcal{L}}(z)$ for all $\langle y\rangle \in(\mathcal{T} E)^{+}, z \in \mathcal{T} \mathcal{L}$. This implies $\tau_{\mathcal{L}} \circ v=$ id.

For the proof that $v$ is filtered and bounded, it suffices to consider the restriction of $v$ to $(d E)^{\text {even }} \odot K$ because $v\left(\left\langle x_{0}\right\rangle d x_{1} \ldots d x_{2 n} \odot k\right)=\left\langle x_{0}\right\rangle \triangleright\left(d x_{1} \ldots d x_{2 n} \triangleright k\right)$. We let $\langle D z\rangle:=D l_{1} \ldots D l_{2 n}$ and $D z:=D l_{2} \ldots D l_{2 n}$ and extend $\alpha$ to a map $\alpha: \mathcal{L}(D \mathcal{L})^{n} \rightarrow \mathcal{L}(D \mathcal{L})^{n+1}$ by $\alpha\left(l_{0} D l_{1} \ldots D l_{2 n}\right):=\alpha\left(l_{0}\right) D l_{1} \ldots D l_{2 n}$. Straightforward computations show that

$$
\begin{aligned}
d x_{1} d x_{2} \triangleright l_{0}\langle D z\rangle= & \left(x_{1} x_{2}\right) \triangleright l_{0}\langle D z\rangle-x_{1} \triangleright\left(x_{2} \triangleright l_{0}\langle D z\rangle\right) \\
= & d x_{1} d x_{2} \odot l_{0}\langle D z\rangle-D \alpha\left(d x_{1} d x_{2} \odot l_{0}\right)\langle D z\rangle \\
& +\alpha\left(x_{1} \odot \alpha\left(x_{2} \odot l_{0}\right)\right)\langle D z\rangle . \\
d x_{1} d x_{2} \triangleright D l_{1} D z=\alpha & \left(\left(x_{1} x_{2}\right) \odot l_{1}\right) D z-x_{1} \odot \alpha\left(x_{2} \odot l_{1}\right) D z \\
& +D \alpha\left(x_{1} \odot \alpha\left(x_{2} \odot l_{1}\right)\right) D z .
\end{aligned}
$$


To prove that $v$ is filtered we construct a filtration $(\mathcal{T L})_{n}^{\prime}$ on $\mathcal{T} \mathcal{L}$ that is equivalent to the standard filtration and for which $d E d E \triangleright(\mathcal{T} \mathcal{L})_{n}^{\prime} \subset(\mathcal{T} \mathcal{L})_{n+1}^{\prime}$ for all $n$. Hence $v\left((d E)^{2 n} \odot K\right) \subset(\mathcal{T} \mathcal{L})_{n}^{\prime}$ for all $n$, so that $v$ is filtered. Define

$$
\#_{e}\left(l_{0} D l_{1} \ldots D l_{2 n}\right):=3 n, \quad \#_{e}\left(D l_{1} \ldots D l_{2 n}\right):=3 n-2,
$$

and

$$
\#_{i}\left(\left\langle l_{0}\right\rangle D l_{1} \ldots D l_{2 n}\right):=\#\left\langle l_{0}\right\rangle+\# l_{1}+\cdots+\# l_{2 n}
$$

for homogeneous $\left\langle l_{0}\right\rangle, l_{1}, \ldots, l_{2 n}$. Define $\#_{t}=\#_{e}+\#_{i}$ and let $(\mathcal{T} \mathcal{L})_{n}^{\prime}$ be the linear span of all homogeneous monomials $z \in \mathcal{T} \mathcal{L}$ with $\#_{t} z \geq n$. We have $\#_{t}\left\langle l_{0}\right\rangle D l_{1} \ldots D l_{2 m}>n$ if $m>n$ or if $\# l_{j}>n$ for some $j$. Conversely, if neither $m>n$ nor $\# l_{j}>n$ for any $j$, then $\#_{t}\left\langle l_{0}\right\rangle D l_{1} \ldots D l_{2 m} \leq 3 n+(2 n+1) n$. Therefore, the filtration $(\mathcal{T} \mathcal{L})_{n}^{\prime}$ is equivalent to the standard filtration.

During the proof of Lemma 4 we observed that $\mu_{5}^{-1}$ decreases \# by at most 1 . Therefore, $\alpha(l)$ is a sum of terms $l_{0} D l_{1}$ with $\# l_{0}+\# l_{1} \geq \# l-1$. Inspecting the summands in (11) and (12), we see that $d x_{1} d x_{2} \triangleright z$ is a sum of homogeneous terms of degree $\#_{t} \sqcup \geq \#_{t} z+1$. The possible loss in $\#_{i}$ through $\alpha$ is compensated by the gain in $\#_{e}$. Consequently, $d E d E \triangleright(\mathcal{T} E)_{n}^{\prime} \subset(\mathcal{T} E)_{n+1}^{\prime}$ as desired.

The boundedness of $v$ is more difficult. We are going to prove the following Lemma:

Lemma 6. For all $T \in \mathfrak{S}(\mathcal{T} \mathcal{L})$ and $S \in \mathfrak{S}(E)$, there is $F \in \mathfrak{S}(\mathcal{T} \mathcal{L})$ with $T \subset F$ and $d S d S \triangleright F \odot F$.

Let $T=S_{K} \subset K$ for some $S_{K} \in \mathfrak{S}(K)$. Replacing the set $F$ of Lemma 6 by $F^{\circ}$, we can achieve that $S_{K} \subset F$ and $d S d S \triangleright F \subset F$. By induction, $(d S)^{2 n} \triangleright F \subset F$ for all $n$, that is, $(d S)^{\text {even }} \triangleright S_{K} \subset F$. Thus Lemma 6 implies that $v$ is bounded.

To prove Lemma 6, we make the Ansatz

$$
F:=F_{0}\left(D F_{\infty}\right)^{\text {even }} \cup D F_{0} D F_{\infty}\left(D F_{\infty}\right)^{\text {even }}
$$

with certain $F_{0}, F_{\infty} \in \mathfrak{S}(\mathcal{L})$ and $\left(D F_{\infty}\right)^{\text {even }}:=\bigcup_{n=0}^{\infty}\left(D F_{\infty}\right)^{2 n} \subset(\mathcal{T} \mathcal{L})^{+}$. Inspecting the summands in (11) and (12), we see that $d S d S \triangleright F \Subset F$ follows if

$$
\begin{array}{cr}
3 d S d S F_{0} \odot F_{0}, & 3 \alpha\left(S \odot \alpha\left(S \odot F_{0}\right)\right) \odot F_{0} D F_{\infty} D F_{\infty}, \\
3 \alpha\left(d S d S F_{0}\right) \odot F_{0} D F_{\infty}, & 3 \alpha\left(S^{2} \odot F_{0}\right) \odot F_{0} D F_{\infty} .
\end{array}
$$

The factors of 3 are needed to convert sums of three terms into convex combinations. Choose $S_{2} \in \mathfrak{S}(E)$ with $2 S \cup(2 S)^{2} \subset S_{2}$. The conditions (14) follow if

$$
d S_{2} d S_{2} F_{0} \odot F_{0}, \quad\left\langle S_{2}\right\rangle \odot \alpha\left(\left\langle S_{2}\right\rangle \odot F_{0}\right) \odot F_{0} D F_{\infty} .
$$

Let $\omega_{s}: Q \times Q \rightarrow K$ be the curvature of the linear section $s: Q \rightarrow E$. We choose $S_{Q} \in \mathfrak{S}(Q)$ and (afterwards) $S_{K} \in \mathfrak{S}(K)$ such that $2 S_{2} \cup 2 S_{2}^{2} \odot S_{E}:=S_{K} \cup s\left(S_{Q}\right)$ 
and $\omega_{S}\left(S_{Q}, S_{Q}\right) \subset S_{K}$. To avoid notational clutter, we will frequently suppress the map $s$ and write $S_{Q}$ instead of $s\left(S_{Q}\right)$. Let $S_{K}^{\prime}:=\left\langle S_{E}\right\rangle \cdot S_{K} \cdot\left\langle S_{E}\right\rangle \in \mathfrak{S}(K)$. Let

$$
F_{0}:=\left(d S_{2}\right)^{\text {even }}\left\langle S_{2}\right\rangle \odot\left(d S_{Q}\right)^{\text {even }}\left\langle S_{Q}\right\rangle \odot S_{K}^{\prime} \in \mathfrak{S}(\mathcal{L}) .
$$

Condition (15) follows if $\alpha\left(\left\langle S_{2}\right\rangle \odot F_{0}\right) \odot\left(d S_{Q}\right)^{\text {even }}\left\langle S_{Q}\right\rangle \odot S_{K}^{\prime} D F_{\infty}$. Since $2 S_{2} \cup 2 S_{2}^{2} \odot S_{E}$, we have $\left\langle S_{2}\right\rangle \odot\left(d S_{2}\right)^{\text {even }}\left\langle S_{2}\right\rangle \Subset\left(d S_{E}\right)^{\text {even }}\left\langle S_{E}\right\rangle$. Hence

$$
\alpha\left(\left(d S_{E}\right)^{\text {even }}\left\langle S_{E}\right\rangle \odot\left(d S_{Q}\right)^{\text {even }}\left\langle S_{Q}\right\rangle \odot S_{K}^{\prime}\right) \odot\left(d S_{Q}\right)^{\text {even }}\left\langle S_{Q}\right\rangle \odot S_{K}^{\prime} D F_{\infty}
$$

implies $d S d S \triangleright F \Subset F$. Let $T^{\prime}:=\left(d S_{E}\right)^{\text {even }}\left\langle S_{E}\right\rangle \cap \mathcal{I}$. We are going to show that the left hand side of (17) is contained in $\alpha\left(T^{\prime} \odot T^{\prime \prime}\right)^{\circ}$ for some small set $T^{\prime \prime} \in \mathfrak{S}(\mathcal{L})$. Since $\left(d S_{E}\right)^{\text {even }}\left\langle S_{E}\right\rangle=\left(d S_{Q}\right)^{\text {even }}\left\langle S_{Q}\right\rangle \cup T^{\prime}$, the only problematic part is $\alpha\left(\left(d S_{Q}\right)^{\text {even }}\left\langle S_{Q}\right\rangle \odot\left(d S_{Q}\right)^{\text {even }}\left\langle S_{Q}\right\rangle \odot S_{K}^{\prime}\right)$. Let

$$
y=d q_{1} \ldots d q_{2 n}\left\langle q_{2 n+1}\right\rangle \odot d q_{2 n+2} \ldots d q_{2 n+2 m-1}\left\langle q_{2 n+2 m}\right\rangle \odot k
$$

with $q_{j} \in S_{Q}=s\left(S_{Q}\right), k \in S_{K}^{\prime}$. We omit the section $s$ whenever this does not create confusion. When bringing $y$ into right handed standard form, we get a sum of terms of the form $\pm d q_{1} \ldots d q_{j-1} d\left(s\left(q_{j}\right) s\left(q_{j+1}\right)\right) d q_{j+2} \ldots$ We can replace $s\left(q_{j}\right) s\left(q_{j+1}\right)=s\left(q_{j} q_{j+1}\right)-\omega_{s}\left(q_{j}, q_{j+1}\right)$ by $-\omega_{s}\left(q_{j}, q_{j+1}\right)$ because $\alpha$ annihilates $d q_{1} \ldots d q_{j-1} d s\left(q_{j} q_{j+1}\right) d q_{j+2} \ldots d q_{2 n+2 m-1}\left\langle q_{2 n+2 m}\right\rangle \odot k \in G$. By assumption, $\omega_{s}\left(q_{j}, q_{j+1}\right) \in \omega_{S}\left(S_{Q}, S_{Q}\right) \subset S_{K}$. Thus we can replace $y$ by a sum of terms in

$$
\left(d S_{Q}\right)^{j-1} d S_{K}\left(d S_{Q}\right)^{2 n+2 m-j-2}\left\langle S_{Q}\right\rangle \odot S_{K}^{\prime}
$$

and a last term (for $j=2 n+2 m-1$ ) in $\left(d S_{Q}\right)^{2 n+2 m-2} S_{K} \odot S_{K}^{\prime}$. Since the geometric series $\sum 2^{-j}$ converges, we can write this sum as a convex combination of terms in

$$
\left(\left(d S_{E}\right)^{\text {even }} \cap \mathcal{I}\right)\left(d 2 S_{Q}\right)^{\text {even }}\left\langle S_{Q}\right\rangle \odot C S_{K}^{\prime} \subset T^{\prime} \odot\left(d 2 S_{Q}\right)^{\text {even }}\left\langle S_{Q}\right\rangle \odot C S_{K}^{\prime}
$$

for a suitable constant $C \geq 1$. Consequently,

$$
\alpha\left(\left(d S_{E}\right)^{\text {even }}\left\langle S_{E}\right\rangle \odot\left(d S_{Q}\right)^{\text {even }}\left\langle S_{Q}\right\rangle \odot S_{K}^{\prime}\right) \odot \alpha\left(T^{\prime} \odot T^{\prime \prime}\right)
$$

with $T^{\prime \prime}:=\left(d 2 S_{Q}\right)^{\text {even }}\left\langle S_{Q}\right\rangle \odot C S_{K}^{\prime}$ as desired.

The map $\mu_{5}^{-1}$ is a right $\mathcal{T} E$-module homomorphism. Therefore and because $T^{\prime} \subset \mathcal{I}$, we have $\mu_{5}^{-1}\left(T^{\prime} \odot T^{\prime \prime}\right)=\mu_{5}^{-1}\left(T^{\prime}\right) \odot T^{\prime \prime}$. Equation (6) implies

$$
\mu_{5}^{-1}\left(T^{\prime} \odot T^{\prime \prime}\right) \odot\left(d S_{Q}\right)^{\text {even }}\left\langle S_{Q}\right\rangle \otimes S_{K}^{\prime} \otimes 2\left\langle S_{E}\right\rangle \odot\left(d S_{E}\right)^{\text {even }}\left\langle S_{E}\right\rangle \odot T^{\prime \prime} .
$$

As a result, we get $\alpha\left(T^{\prime} \odot T^{\prime \prime}\right) \odot\left(d S_{Q}\right)^{\text {even }}\left\langle S_{Q}\right\rangle \odot S_{K}^{\prime} D F_{\infty}$ with

$$
\begin{aligned}
F_{\infty}:=2\left\langle S_{E}\right\rangle \odot( & \left(d S_{E}\right)^{\text {even }}\left\langle S_{E}\right\rangle \odot T^{\prime \prime} \\
& =2 C\left\langle S_{E}\right\rangle \odot\left(d S_{E}\right)^{\text {even }}\left\langle S_{E}\right\rangle \odot\left(d 2 S_{Q}\right)^{\text {even }}\left\langle S_{Q}\right\rangle \odot S_{K}^{\prime} .
\end{aligned}
$$

Consequently, sets $F$ of the form (13) with $F_{0}$ as in (16) and $F_{\infty}$ as in (18) satisfy $d S d S \triangleright F \odot F$. Any small subset $T \subset \mathcal{T} \mathcal{L}$ is contained in the disked hull of $F$ for suitably big $S_{2} \in \mathfrak{S}(E)$. Therefore, Lemma 6 is true, so that $v$ is bounded. 


\subsection{Homotopy equivalence of $\mathcal{L}$ and $\mathcal{T} K$}

Let $\kappa: \mathcal{L} \rightarrow K$ be the restriction of $\tau_{E}: \mathcal{T} E \rightarrow E$ to $\mathcal{L}$. We claim that

$$
X(\mathcal{T} \kappa) \circ X(v): X(\mathcal{L}) \rightarrow X(\mathcal{T} \mathcal{L}) \rightarrow X(\mathcal{T} K)
$$

is a homotopy inverse for $X(j): X(\mathcal{T} K) \rightarrow X(\mathcal{L})$. We have $(\mathcal{T} \kappa) \circ v \circ j=$ $\operatorname{id}_{\mathcal{T} K}$ because this holds on the subspace $\sigma(K) \subset \mathcal{T} K$ generating $\mathcal{T} K$. Hence $X(\mathcal{T} \kappa) \circ X(v)$ is a section for $X(j)$. We are going to show that the homomorphisms $j \circ \mathcal{T} \kappa: \mathcal{T} \mathcal{L} \rightarrow \mathcal{L}$ and $\tau_{\mathcal{L}}: \mathcal{T} \mathcal{L} \rightarrow \mathcal{L}$ are polynomially homotopic.

Define a linear map $h: \mathcal{L} \rightarrow \mathbb{C}[t] \otimes \mathcal{L}$ by $h(l):=t^{\# l} \otimes l$ for homogeneous $l$. Its curvature $\omega_{h}\left(l_{1}, l_{2}\right):=h\left(l_{1} \odot l_{2}\right)-h\left(l_{1}\right) \odot h\left(l_{2}\right)$ is given by

$$
\begin{aligned}
& \omega_{h}\left(l_{1}, l_{2}\right)=h\left(l_{1} \cdot l_{2}-d l_{1} d l_{2}\right)-h\left(l_{1}\right) \cdot h\left(l_{2}\right)+d h\left(l_{1}\right) \cdot d h\left(l_{2}\right) \\
& \quad=t^{\# l_{1}+\# l_{2}}\left(l_{1} \cdot l_{2}-t d l_{1} d l_{2}-l_{1} \cdot l_{2}+d l_{1} d l_{2}\right)=t^{\# l_{1}+\# l_{2}}(1-t) \otimes d l_{1} d l_{2}
\end{aligned}
$$

for homogeneous $l_{1}, l_{2} \in \mathcal{L}$. The universal property of the tensor algebra $\mathcal{T} \mathcal{L}$ allows us to extend $h$ to a homomorphism $H: \mathcal{T} \mathcal{L} \rightarrow \mathbb{C}[t] \otimes \mathcal{L}$ by

$$
\begin{aligned}
H\left(\left\langle l_{0}\right\rangle D l_{1} \ldots D l_{2 n}\right)=h\left\langle l_{0}\right\rangle \odot & \omega_{h}\left(l_{1}, l_{2}\right) \odot \cdots \odot \omega_{h}\left(l_{2 n-1}, l_{2 n}\right) \\
& =t^{\#\left\langle l_{0}\right\rangle+\# l_{1}+\cdots+\# l_{2 n}}(1-t)^{n} \otimes\left\langle l_{0}\right\rangle d l_{1} \ldots d l_{2 n} .
\end{aligned}
$$

The map $H_{0}=\operatorname{ev}_{0} \circ H$ annihilates $\left\langle l_{0}\right\rangle D l_{1} \ldots D l_{2 n}$ if $\# l_{j}>0$ for some $j$, whereas $H_{1}=\mathrm{ev}_{1} \circ H$ annihilates $\left\langle l_{0}\right\rangle D l_{1} \ldots D l_{2 n}$ if $n>0$. Consequently, $H_{0}=j \circ \mathcal{T} \kappa$ and $H_{1}=\tau_{\mathcal{L}}$. The map $H$ is filtered and bounded with respect to the bornology and filtration on $\mathbb{C}[t] \otimes \mathcal{L}$ defined in Sect. 4.2. Hence $j \circ \mathcal{T}_{\kappa}$ and $\tau_{\mathcal{L}}$ are polynomially homotopic. It follows that $j \circ(\mathcal{T} \kappa) \circ v$ is polynomially homotopic to $\mathrm{id}_{\mathcal{L}}$. Therefore, the algebras $\mathcal{L}$ and $\mathcal{T} K$ are homotopy equivalent.

Prop. 2 yields that $X\left(j \circ \mathcal{T}_{\kappa}\right)$ and $X\left(\tau_{\mathcal{L}}\right)$ are chain homotopic. As a result, $X(j) \circ X(\mathcal{T} \kappa) \circ X(v) \sim X\left(\tau_{\mathcal{L}}\right) \circ X(v)=$ id because $\tau_{\mathcal{L}} \circ v=\operatorname{id}_{\mathcal{L}}$. Thus $X(j)$ is a homotopy equivalence. The map $\psi: X(\mathcal{L}) \rightarrow X(\mathcal{T} E: \mathcal{T} Q)$ is a homotopy equivalence as well by Lemma 2. Consequently, $\varrho=\psi \circ X(j)$ is a homotopy equivalence. The proof of the excision theorem is complete.

\section{Excision results in cyclic cohomology}

We can describe $\operatorname{HP}^{*}(A)$ as the inductive limit of the cyclic cohomology groups $\mathrm{HC}^{*+2 n}(A)$. We may ask how the connecting map $\partial: \mathrm{HP}^{*}(K) \rightarrow \mathrm{HP}^{*-1}(Q)$ shifts the dimensions in cyclic cohomology. Puschnigg was the first to show that $\partial$ maps $\mathrm{HC}^{n}(K)$ to $\mathrm{HC}^{3 n+3}(Q)$. He also proves that this estimate is optimal for certain extensions [11]. The proof of excision above yields, in principle, an explicit formula for a degree 1 chain map $\delta: X(\mathcal{T} Q) \rightarrow X(\mathcal{T} K)$ that induces the boundary map in the six term exact sequences. Messy bookkeeping in [10] shows that the map on cohomology induced by $\delta$ maps $\operatorname{HC}^{n}(K)$ to $\mathrm{HC}^{3 n+3}(Q)$ and thus realizes the optimal bound. [10] also contains estimates about the dimension shifts that occur when switching between $\mathrm{HC}^{*}(K)$ and the relative cyclic cohomology $\mathrm{HC}^{*}(E: Q)$. 


\section{References}

1. A. Connes (1988): Entire cyclic cohomology of Banach algebras and characters of $\theta$-summable Fredholm modules. $K$-Theory $1(6), 519-548$

2. A. Connes (1994): Noncommutative geometry. San Diego, CA: Academic Press Inc.

3. J. Cuntz (1997): Excision in periodic cyclic theory for topological algebras. In: Cyclic cohomology and noncommutative geometry (Waterloo, ON, 1995), pp. 43-53. Providence, RI: Amer. Math. Soc.

4. J. Cuntz, D. Quillen (1995): Algebra extensions and nonsingularity. J. Amer. Math. Soc. 8(2), 251-289

5. J. Cuntz, D. Quillen (1995): Cyclic homology and nonsingularity. J. Amer. Math. Soc. 8(2), 373-442

6. J. Cuntz, D. Quillen (1997): Excision in bivariant periodic cyclic cohomology. Invent. math. 127(1), 67-98

7. H. Hogbe-Nlend (1970): Complétion, tenseurs et nucléarité en bornologie. J. Math. Pures Appl. (9) 49, 193-288

8. H. Hogbe-Nlend (1977): Bornologies and functional analysis. Amsterdam: NorthHolland Publishing Co.

9. M. Khalkhali (1994): On the entire cyclic cohomology of Banach algebras. Comm. Algebra 22(14), 5861-5874

10. R. Meyer (1999): Analytic cyclic cohomology. Westfälische Wilhelms-Universität Münster. URL http://xxx.lanl.gov/abs/math.KT/9906205

11. M. Puschnigg (2001): Excision in cyclic homology theories. Invent. math. 143(2), 249-323

12. M. Wodzicki (1989): Excision in cyclic homology and in rational algebraic $K$-theory. Ann. of Math. (2) 129(3), 591-639 